\title{
Kinetics of Sorption of Uranium(VI) Compounds with Zirconium-Silica Nanosorbents
}

\author{
O. V. Perlova ${ }^{a}$, V. F. Sazonova ${ }^{a}$, N. A. Perlova ${ }^{a}$, and N. A. Yaroshenko ${ }^{b}$ \\ ${ }^{a}$ Odessa National University, Odessa, Ukraine \\ ${ }^{b}$ Pisarzhevskii Institute of Physical Chemistry, National Academy of Sciences of Ukraine, Kyiv, Ukraine \\ e-mail:olga_perlova@onu.edu.ua \\ Received February 10, 2013
}

\begin{abstract}
The kinetics of sorption of uranium(VI) compounds from sulfate and carbonate solutions using four samples of mesoporous zirconium-silica nanosorbents obtained by bitemplate (solubilization) synthesis was studied. The sorption equilibrium set-in time and the kinetic characteristics of sorption were shown to depend on the sorbent (its composition, specific surface area, dispersity, and pore size), the temperature, and the composition and $\mathrm{pH}$ of the solution from which uranium compounds are sorbed. The sorption kinetics was described by a first-order equation. The limiting stage of the process was found to be the external diffusion of uranium-containing particles to the sorbent surface.
\end{abstract}

Keywords: kinetics, sorption, zirconium-silica nanosorbents, uranium(VI) compounds.

DOI: $10.1134 / \mathrm{S} 0036024414060223$

\section{INTRODUCTION}

Selective extraction of uranium compounds from complex mixtures including natural and waste waters is very important in nuclear power engineering and other uranium ore processing industries. This problem became especially acute in view of the necessity of Chernobyl emergency management. Deactivation of environmental objects and natural and industrial waters is necessary for environmental improvement. Sorption is one of the effective methods of deactivation (removal of uranium compounds from aqueous media).

As is known, studies of the kinetics of sorption of solutes on solid sorbents give useful information about the sorption mechanism [1-3]. This allows optimization of sorption and synthesis conditions of sorbents with specified (for particular systems) properties. In particular, this concerns silica sorbents, which are finding increasing use in extraction of toxic and valuable components from aqueous media.

Silica nanosorbents are characterized by definite advantages over other industrial mineral sorbents (alumina, zeolites, etc.): low temperature $\left(110-200^{\circ} \mathrm{C}\right)$ required for sorbent regeneration and hence low energy consumption, the possibility of synthesizing silica nanosorbents in a wide range of specified structural characteristics using simple procedures, and high abrasion and compression stability.

The goal of this study was to investigate the sorption kinetics of uranium(VI) compounds with new zirconium-silica nanosorbents.

\section{EXPERIMENTAL}

The objects of investigation were solutions of uranyl acetate containing $2 \times 10^{-4} \mathrm{~mol}$ of uranium(VI) and $2 \times 10^{-2} \mathrm{~mol}$ of carbonate or sulfate ions per liter. The solutions were prepared from reagent-grade $\mathrm{UO}_{2}\left(\mathrm{CH}_{3} \mathrm{COO}\right)_{2} \cdot 2 \mathrm{H}_{2} \mathrm{O}, \mathrm{NaHCO}_{3}$, and $\mathrm{H}_{2} \mathrm{SO}_{4}$. The initial $\mathrm{pH}$ of the uranium(VI) carbonate and sulfate solutions was 8 and 2 . Under the given experimental conditions, uranium was in the form of the anion complexes $\left[\mathrm{UO}_{2}\left(\mathrm{CO}_{3}\right)_{2}\right]^{2-}(87 \%)$ and $\left[\mathrm{UO}_{2}\left(\mathrm{CO}_{3}\right)_{3}\right]^{4-}$ $(13 \%)$ in the carbonate solutions and in the form of a mixture of $\mathrm{UO}_{2}^{2+}(46.3 \%)$, $\left[\mathrm{UO}_{2}\left(\mathrm{SO}_{4}\right)\right](46.3 \%)$, and $\left[\mathrm{UO}_{2}\left(\mathrm{SO}_{4}\right)_{2}\right]^{2-}(6.5 \%)$ in the sulfate solutions [4]. Experiments on the kinetics of sorption of uranium compounds from carbonate and sulfate solutions were performed at initial and experimentally established [5] optimum $\mathrm{pH}$ values, namely, $\mathrm{pH} 4$ (starting carbonate solutions) and 7 (sulfate solutions). In the starting carbonate solutions with $\mathrm{pH} 4$, uranium(VI) was in the form of a mixture of $\mathrm{UO}_{2}^{2+}(91 \%)$ and $\mathrm{UO}_{2} \mathrm{OH}^{+}(9 \%)$ cations; in sulfate solutions with $\mathrm{pH} 7$, as a mixture of $\mathrm{UO}_{2} \mathrm{OH}^{+}(13.7 \%)$ and $\mathrm{UO}_{2}(\mathrm{OH})_{2}(86.3 \%)$ [4].

As the sorbents, we used the experimental samples of mesoporous zirconium-silica nanosorbents synthesized by the bitemplate (solubilization) procedure from inexpensive reagents: sodium silicate, zirconyl chloride, and the industrial fraction of surfactant $[6$, 7]. The starting freshly synthesized sorbent samples were subjected to hydrothermal $\left(80^{\circ} \mathrm{C}\right)$ and thermal $\left(600^{\circ} \mathrm{C}\right)$ treatments. The specific surface area of samples 1-4 (Table 1) was determined from argon sorption by the BET method [8] using the data of the isotherms of low-temperature sorption-desorption of 
Table 1. Characteristics of zirconium-silica sorbents used

\begin{tabular}{c|c|c|c|c|c}
\hline Sample & $S_{\mathrm{sp}}, \mathrm{m}^{2} / \mathrm{g}$ & $W\left(\mathrm{ZrO}_{2}\right), \%$ & $d_{\text {pore }}, \mathrm{nm}$ & $r, \mu \mathrm{m}$ & $\mathbf{r}_{\mathrm{av}}, \mu \mathrm{m}$ \\
\hline 1 & 1300 & 24 & 3.18 & $2-76$ & 6.4 \\
2 & 1020 & 29 & & $0.4-56$ & 4.4 \\
3 & 900 & 37 & & $2-52$ & 8.5 \\
4 & 700 & 37 & 7.59 & $2-120$ & 5.5 \\
\hline
\end{tabular}

$S_{\mathrm{sp}}$ is the specific surface area, $W$ is the mass fraction of $\mathrm{ZrO}_{2}, d_{\text {pore }}$ is the pore diameter, $r$ is the particle radius, and $\mathbf{r}_{\mathrm{av}}$ is the average particle radius.

nitrogen (samples 1 and 4) obtained on a Quantachrome NovaWin2 instrument. The size distribution of pores was determined from the desorption isotherms using the $\mathrm{BJH}$ model. When the mass fraction of $\mathrm{ZrO}_{2}$ in the sorbent samples increased, the pore diameter of the sorbents increased, while the specific surface area accordingly decreased (Table 1 ).

The range of $\mathrm{pH}$ corresponding to the isoelectric state of the sorbent surface was 3.0-3.5 [5].

Experiments on uranium sorption with the sorbents were performed under the static conditions in the following way. The sorbent $(0.03 \mathrm{~g})$ was placed in a conic $100 \mathrm{~cm}^{3}$ flask and the solution under study $\left(75 \mathrm{~cm}^{3}\right)$ was poured on it. The flask was stoppered and allowed to stay at $20 \pm 2,30 \pm 2$, or $40 \pm 2^{\circ} \mathrm{C}$ for $0.5-$ $8 \mathrm{~h}$. The flask contents were then subjected to ultrafiltration through parchment ultrafilters preliminarily soaked in distilled water for $45-50 \mathrm{~min}$. The filtrate was analyzed for uranium content photocolorimetrically by the standard procedure [9] using the arsenazo III reagent at a wavelength of $670 \mathrm{~nm}$ with an absorbing layer thickness of $1 \mathrm{~cm}$.

According to the experiment, the sorption of solutes on solid sorbents is generally described by the first-order kinetic equation, which indicates that the limiting stage is diffusion of particles from the bulk of the solution to the surface of the sorbent and then to its pores. Therefore, to describe the soprtion kinetics, we used the equation

$$
\ln \frac{C}{C_{0}}=-K t
$$

where $C$ is the uranium concentration in solution at a moment of time $t\left(\mathrm{~mol} / \mathrm{dm}^{3}\right), C_{0}$ is the initial uranium concentration in solution $\left(\mathrm{mol} / \mathrm{dm}^{3}\right)$, and $K$ is the rate constant of sorption $\left(\mathrm{s}^{-1}\right)$.

The $K$ constant was determined by graphically solving Eq. (1).

To describe the external diffusion, we used a simplified approximation [10], which suggests that at the initial moment of phase contact, the concentration of the substance on the sorbent surface is zero and diffusion inside the particle is ignored:

$$
\ln \frac{C}{C_{0}}=-k \frac{A}{V} t
$$

where $k$ is the external mass transfer coefficient $(\mathrm{m} / \mathrm{s})$, $A$ is the sorbent exchange surface $\left(\mathrm{m}^{2}\right)$, and $V$ is the volume of the solution $\left(\mathrm{m}^{3}\right)$.

The $A / V$ ratio was calculated by the formula

$$
\frac{A}{V}=\frac{S_{\mathrm{sp}} m}{V}
$$

where $m$ is the sorbent sample $(\mathrm{g})$, and $S_{\mathrm{sp}}$ is the specific surface area of the sorbent $\left(\mathrm{m}^{2} / \mathrm{g}\right)$.

The external diffusion was described using the equation suggested by Boyd, Adamson, and Myers [11]

$$
F=\frac{S}{S_{\infty}}=1-\frac{6}{\pi^{2}} \sum_{n=1}^{\infty} \frac{1}{n^{2}} \exp \left(-\frac{D \pi^{2} n^{2} t}{r_{0}^{2}}\right),
$$

where $D$ is the inner diffusion coefficient $\left(\mathrm{m}^{2} \mathrm{~s}^{-1}\right) ; r_{0}$ is the mean radius of sorbent particles (m); $S$ and $S_{\infty}$ are the degree of sorption at a moment of time $t$ and at equilibium (\%); $F$ is the degree of conversion, which depends only on the parameter $B=D \pi^{2} / r_{0}^{2}\left(\mathrm{~s}^{-1}\right)$; and $n$ is the series of integers $(1,2,3,4$, etc.).

At high degrees of conversion $(F>0.7)$, the higherorder terms in (4) become small, leading to a simplification of the equation:

$$
F=\frac{S}{S_{\infty}}=1-\frac{6}{\pi^{2}} \exp (-B t)
$$

or

$$
\ln (1-F)=\ln \frac{6}{\pi^{2}}-B t
$$

To determine $D$, we constructed a plot in semilogarithmic coordinates $\ln (1-F)=f(t)$. $B$ was determined from the slope of the line relative to the abscissa; then $D$ was calculated.

The average radii of sorbent particles were determined by investigating their size distribution according to the micrographs obtained with a Sigeta MB-508 microscope equipped with a Goryaev counting chamber and linked to a computer. The average radius of sorbent particles was calculated by the equation [12]

$$
\mathbf{r}_{\mathrm{av}}=\sum n_{i} r_{i} / \sum n_{i}
$$

where $n_{i}$ is the content of particles with a radius $r_{i}$ in a given fraction.

The experimental and calculated data given in Table 1 were used for calculating the kinetic parameters of sorption.

The $\mathrm{pH}$ of solutions was measured using a universal EV-74 ion meter with a glass electrode. $\mathrm{pH}$ of the solutions was adjusted using 0.1 and $1.0 \mathrm{M} \mathrm{KOH}$ and $\mathrm{HCl}$ solutions.

\section{RESULTS AND DISCUSSION}

Our studies showed (Fig. 1, Tables 2 and 3) that the sorption equilibrium set-in time and the kinetic characteristics of sorption depend on the nature of the sorbent sample (its composition, specific surface area, dispersity, and pore size), $\mathrm{pH}$ and composition 

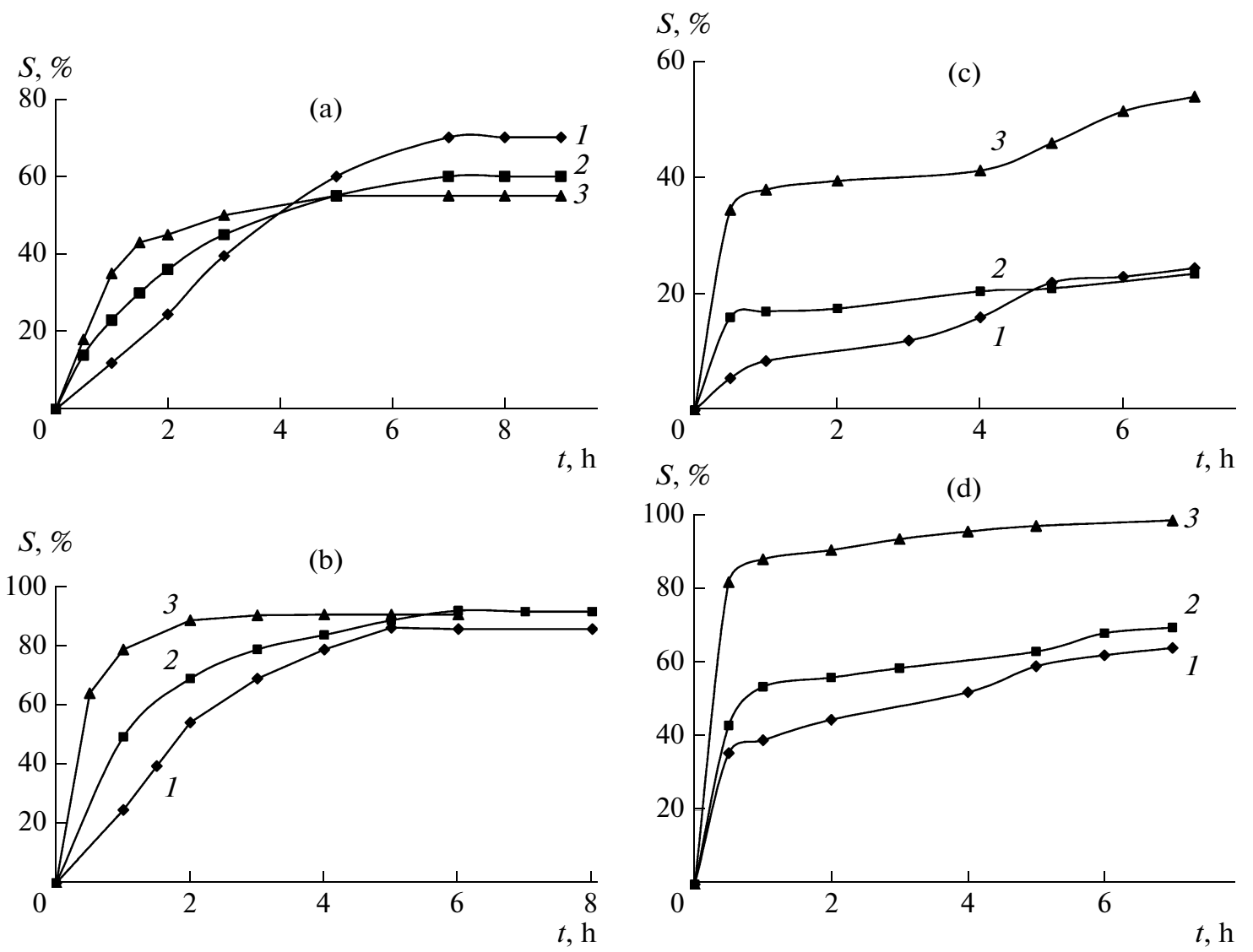

Fig. 1. Effect of time $(t)$ on the degree of uranium sorption $(S)$ from carbonate solutions with pH (a) 8 and (b) 4 and sulfate solutions with pH (c) 2 and (d) 7 with sorbent 2 at temperatures of (1) 293, (2) 303, and (3) $313 \mathrm{~K}$.

of the solution from which uranium is sorbed, and temperature.

When uranium compounds were extracted from carbonate solutions with $\mathrm{pH} 8$, the maximum sorption rate constant was observed for sample 4 with a specific surface area of $700 \mathrm{~m}^{2} / \mathrm{g}$ and a pore diameter of $7.59 \mathrm{~nm}$. When sorbent 1 with pores of smaller diameter $(3.18 \mathrm{~nm})$ was used, the reaction rate constant was 1.5-3.3 times lower than for sample 4. The 1.52.0-fold lower inner diffusion coefficients in this case were evidently related to steric hindrances, which generally appear during the sorption of large, highly charged uranyl bi- and tricarbonate complexes in the pores of sorbent 1 .

In contrast, when uranium compounds were extracted from sulfate solutions with $\mathrm{pH} 2$, the kinetic characteristics of the process with samples 1 and 4 differed but slightly (Table 3 ). This obviously suggests that steric factors are less significant during the sorption of uranyl cations and uranyl sulfate complexes.

During the sorption of uranium compounds from sulfate and carbonate solutions with initial $\mathrm{pH}$ values, the inner diffusion coefficients were the highest when sample 3 was used as a sorbent. It was assumed that the pore size of this sorbent was larger than that of sample 4, which facilitated the inner diffusion of uranyl compounds.

The apparent activation energies of sorption of uranium compounds with the sorbents under study were determined by graphically solving the Arrhenius equation: $27.4-81.5 \mathrm{~kJ} / \mathrm{mol}$ (sulfate solutions) and $16.8-$ $64.6 \mathrm{~kJ} / \mathrm{mol}$ (carbonate solutions) (Tables 2 and 3 ). During the sorption from carbonate solutions with the starting $\mathrm{pH}$ using sorbent samples with increasing average radii, the apparent activation energy decreases and hence the rate constant increases.

For the experimental determination of the limiting stage of sorption, we used a simple and reliable interruption method or the kinetic memory method [11]. In this procedure, sorption was performed for $2 \mathrm{~h}$, after which the solid phase was separated from the uranium(VI) solution. The sorbent and solution were stored separately for $3 \mathrm{~h}$, whereupon the phases were again allowed to react. In the general case for the intradiffusion kinetics, the initial sorption rate was higher than before the interval because the sorbate concentration in the sorbent particle is equalized and its initial gradient increases with the inner diffusion rate. However, it follows from Fig. 2 that a change in the experimental conditions (with and without inter- 
Table 2. Kinetic characteristics of uranium sorption from carbonate solutions

\begin{tabular}{|c|c|c|c|c|c|c|c|c|}
\hline $\mathrm{pH}$ & $T, \mathrm{~K}$ & $K \times 10^{5}, \mathrm{~s}^{-1}$ & $R^{2}$ & $k \times 10^{10}, \mathrm{~m} / \mathrm{s}$ & $t_{\max }, \mathrm{h}$ & $D \times 10^{16}, \mathrm{~m}^{2} / \mathrm{s}$ & $B \times 10^{5}, \mathrm{~s}^{-1}$ & $E, \mathrm{~kJ} / \mathrm{mol}$ \\
\hline \multicolumn{9}{|c|}{ Sorbent 1} \\
\hline \multirow[t]{3}{*}{8} & 293 & 1.83 & 0.997 & 0.35 & 4 & 6.54 & 15.79 & 47.9 \\
\hline & 303 & 4.52 & 0.996 & 0.87 & 4 & 8.85 & 21.30 & \\
\hline & 313 & 6.37 & 0.985 & 1.23 & 2.5 & 8.46 & 20.36 & \\
\hline \multicolumn{9}{|c|}{ Sorbent 2} \\
\hline \multirow[t]{3}{*}{4} & 293 & 11.97 & 0.993 & 2.93 & 5 & 3.92 & 19.88 & 27.6 \\
\hline & 303 & 14.72 & 0.984 & 3.61 & 5 & 2.31 & 11.74 & \\
\hline & 313 & 30.55 & 0.989 & 7.49 & 2 & - & - & \\
\hline \multirow[t]{3}{*}{8} & 293 & 3.83 & 0.991 & 0.94 & 7 & 1.81 & 7.87 & 16.8 \\
\hline & 303 & 7.25 & 0.985 & 1.77 & 7 & 2.43 & 12.06 & \\
\hline & 313 & 7.65 & 0.990 & 1.88 & 6 & 1.94 & 9.85 & \\
\hline \multicolumn{9}{|c|}{ Sorbent 3} \\
\hline \multirow[t]{3}{*}{8} & 293 & 0.87 & 0.939 & 0.24 & 6 & 12.21 & 17.06 & 64.6 \\
\hline & 303 & 2.20 & 0.943 & 0.61 & 6 & 46.82 & 65.42 & \\
\hline & 313 & 4.72 & 0.944 & 1.31 & 4.5 & 28.50 & 39.82 & \\
\hline \multicolumn{9}{|c|}{ Sorbent 4} \\
\hline \multirow[t]{3}{*}{8} & 293 & 6.21 & 0.986 & 1.94 & 5 & 11.08 & 36.11 & 19.6 \\
\hline & 303 & 8.14 & 0.986 & 2.54 & 3.5 & 10.91 & 35.56 & \\
\hline & 313 & 10.37 & 0.978 & 3.24 & 3.5 & 9.37 & 30.54 & \\
\hline
\end{tabular}

$K$ is the sorption rate constant, $k$ is the external mass transfer coefficient, $t_{\max }$ is the time of set-in of sorption equilibrium, $D$ is the inner diffusion coefficient, $B$ is the inner diffusion parameter, $E$ is the apparent activation energy, and $R^{2}$ is the linear correlation coefficient.

Table 3. Kinetic characteristics of uranium sorption from sulfate solutions

\begin{tabular}{|c|c|c|c|c|c|c|c|c|}
\hline $\mathrm{pH}$ & $T, \mathrm{~K}$ & $K \times 10^{5}, \mathrm{~s}^{-1}$ & $R^{2}$ & $k \times 10^{10}, \mathrm{~m} / \mathrm{s}$ & $t_{\max }, \mathrm{h}$ & $D \times 10^{16}, \mathrm{~m}^{2} / \mathrm{s}$ & $B \times 10^{5}, \mathrm{~s}^{-1}$ & $E, \mathrm{~kJ} / \mathrm{mol}$ \\
\hline \multicolumn{9}{|c|}{ Sorbent 1} \\
\hline \multirow[t]{4}{*}{2} & 293 & 3.35 & 0.991 & 0.71 & 4.5 & 3.46 & 8.34 & 27.4 \\
\hline & 303 & 6.26 & 0.986 & 1.33 & 4 & 3.17 & 7.64 & \\
\hline & 313 & 6.84 & 0.935 & 1.45 & 3.5 & 1.28 & 3.08 & \\
\hline & \multicolumn{8}{|c|}{ Sorbent 2} \\
\hline \multirow[t]{3}{*}{2} & 293 & 1.69 & 0.939 & 0.41 & 6 & 2.81 & 14.33 & 81.5 \\
\hline & 303 & 5.18 & 0.938 & 1.26 & 5 & 1.42 & 7.24 & \\
\hline & 313 & 13.28 & 0.999 & 3.23 & 4 & 1.09 & 5.56 & \\
\hline \multirow[t]{3}{*}{7} & 293 & 2.9 & 0.940 & 0.71 & 6 & 4.12 & 20.98 & 48.9 \\
\hline & 303 & 3.4 & 0.994 & 0.83 & 5 & 2.01 & 10.21 & \\
\hline & 313 & 10.5 & 0.935 & 2.57 & 3 & 2.74 & 13.97 & \\
\hline \multicolumn{9}{|c|}{ Sorbent 3} \\
\hline \multirow[t]{3}{*}{2} & 293 & 2.92 & 0.964 & 0.91 & 6 & 54.96 & 75.01 & 29.7 \\
\hline & 303 & 3.73 & 0.961 & 1.16 & 4.5 & 24.43 & 33.37 & \\
\hline & 313 & 6.39 & 0.989 & 2.00 & 4 & 16.28 & 22.24 & \\
\hline \multicolumn{9}{|c|}{ Sorbent 4} \\
\hline \multirow[t]{3}{*}{2} & 293 & 3.91 & 0.964 & 1.22 & 5 & 12.78 & 41.65 & 34.2 \\
\hline & 303 & 5.72 & 0.968 & 1.79 & 5 & 5.97 & 19.46 & \\
\hline & 313 & 8.89 & 0.988 & 2.78 & 4.5 & 5.71 & 18.61 & \\
\hline
\end{tabular}




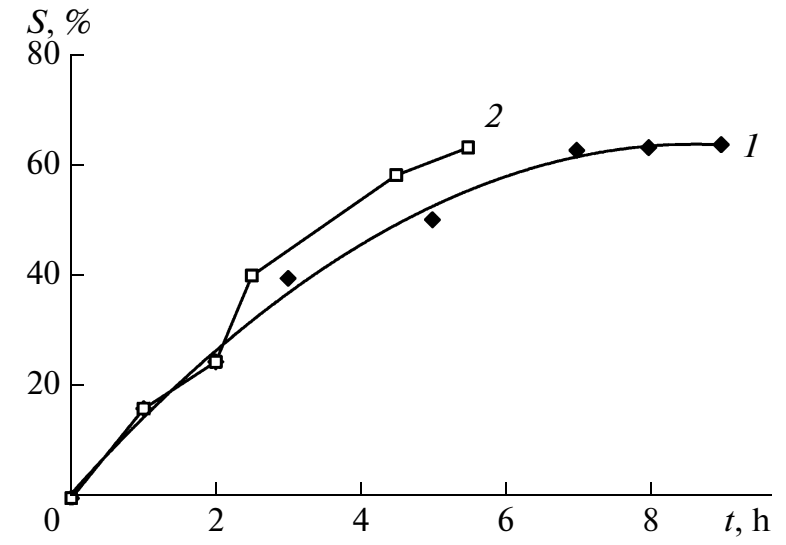

Fig. 2. Effect of time $(t)$ on the degree of uranium sorption $(S)$ from carbonate solutions with pH 8 at $293 \mathrm{~K}$ with sorbent 2 with the process (1) interrupted and (2) not interrupted.

ruption) affects the uranium sorption rate with sorbent 2 insignificantly. This suggests that the role of inner diffusion in the process is insignificant.

Tables 2 and 3 present the external mass transfer coefficients of uranium at $F<0.7$ and inner diffusion coefficients of uranium at $F>0.7$.

The calculated data confirm the outer-diffusion character of the sorption kinetics of uranium compounds because the sorption rate constants $K$ that characterize the outer diffusion are smaller than the $B$ coefficients that characterize the inner diffusion (Tables 2 and 3 ) and the numerical values of the apparent activation energy of the process correspond to the outer diffusion region for systems with a liquid mobile phase. Moreover, there is no strict relationship between the effect of the sorbent particle size (Table 1) on the inner diffusion coefficient (Tables 2 and 3 ) and the effect of the sorbent particle size (Table 1) on the time required for reaching a certain degree of completion of sorption (Tables 2 and 3). This is indirect evidence in favor of the outer diffusion character of sorption.

According to Tables 2 and 3, the kinetic characteristics of uranium sorption from solutions with optimum $\mathrm{pH}$ are better than those of uranium sorption from solutions with initial $\mathrm{pH}$ values. The data obtained can be explained by the difference in the sorbate particle size at optimum and initial $\mathrm{pH}$, which affects the density of their packing in the pores of the sorbent, the difference in the diffusion coefficients, and the negative effect of repulsion forces between the sorbate and sorbent during uranium sorption from solutions with the initial $\mathrm{pH}$ values.

\section{CONCLUSIONS}

Thus as a result of this study, the mechanism of sorption of uranium(VI) compounds from carbonate and sulfate media with zirconium-silica nanosorbents was established. It was shown that the sorption kinetics is described by a first-order equation and the limiting stage is external diffusion of uranium-containing particles to the sorbent surface. The kinetic characteristics of sorption of uranium(VI) compounds and apparent activation energies of sorption can be used for optimizing the procedure for the preparation of zirconium-silica nanosorbents and the conditions of sorption of uranium compounds on them.

\section{REFERENCES}

1. L. A. Pimneva and E. L. Nesterova, Usp. Sovrem. Estestvozn., No. 4, 24 (2008).

2. I. A. Kovalev, N. M. Sorokina, and G. I. Tsizin, Vestn. Mosk. Univ. 41, 309 (2000).

3. E. V. Venetsianov and R. N. Rubenshtein, Dynamics of Sorption from Liquid Media (Nauka, Moscow, 1983) [in Russian].

4. O. V. Perlova and A. A. Shirykalova, J. Water Chem. Technol. 30, 215 (2008).

5. N. A. Yaroshenko, V. F. Sazonova, O. V. Perlova, et al., Russ. J. Appl. Chem. 85, 849 (2012).

6. N. A. Yaroshenko and V. G. Il'in, Russ. J. Appl. Chem. 77, 1769 (2004).

7. S. I. Trofimenko, N. N. Tsyba, and N. A. Yaroshenko, in Proceedings of the Khar'kov Nanotechnical Assembly (Kharkov, 2008), p. 130.

8. Experimental Methods in Adsorption and Molecular Chromatography, Ed. by V. A. Kiselev and V. P. Dreving (Mosk. Gos. Univ., Moscow, 1973) [in Russian].

9. Uranium, Methods for Its Determination, Ed. by V. K. Markov (Atomizdat, Moscow, 1964) [in Russian].

10. G. E. Boyd, A. W. Adamson, and L. S. Myers, J. Am. Chem. Soc. 69, 2836 (1947).

11. G. N. Vol'dman and A. N. Zelikman, Theory of Hydrometallurgical Processes (Intermet Inzhiniring, Moscow, 2003) [in Russian].

12. Laboratory Works and Problems of Colloid Chemistry, Ed. by Yu. G. Frolov and A. S. Grodskii (Khimiya, Moscow, 1986) [in Russian].

Translated by L. Smolina 